\title{
DIAGNOSIS WITH CAUSALITY RELATIONSHIPS AND DIRECTED PATHS IN PN MODELS
}

\author{
Dimitri Lefebvre, Catherine Delherm \\ Université Le Havre - GREAH, 25 rue P. Lebon, 76063 Le Havre, France \\ \{dimitri.lefebvre;catherine.delherm\}@univ-lehavre.fr
}

\begin{abstract}
Petri nets are a suitable tool for the diagnosis of discrete event systems. For this purpose, faulty behaviours are modelled by the firing of failure transitions. This paper is about structural sensitivity in Petri net with respect to the firing of the failure transitions. Algebraic results are provided to characterise the influence and dependence areas of the failure transitions and diagnosability of the systems is obtained as a consequence. The main advantage of our approach is to investigate the diagnosability without working out the marking tree of the diagnoser. Copyright (C2005 IFAC
\end{abstract}

Keywords: Petri nets, structural properties, sensitivity analysis, estimation, diagnosis.

\section{INTRODUCTION}

Fault detection and isolation are important issues for discrete event systems (DES) (Cassandras, 1993). Pioneer applications of the Petri nets (PN) supervisory control in fault detection have been developed that consider faults as forbidden states (Krogh, et al.,1991). The observation of the state was further investigated in order to design controllers with forbidden marking specifications (Giua, et al,. 2002). Another approach to study DES with faulty behaviours concerns PN models with "failure" transitions (Ushio, et al., 1998). In that case, faults are represented with "failure" transitions and faulty behaviours are modelled as firing sequences including some "failure" transitions. The problem consists to detect and isolate the firing of the "failure" transitions that cannot be directly measured. This article focuses on the second approach. The main contribution is to provide some tools useful to decide if a set of observable places and transitions is necessary or sufficient to detect and isolate the firing of "failure" transitions in unobservable firing sequences. For that purpose, influence and dependence areas of the "failure" transitions are investigated according to the directed paths and causality relationships in PN models. Our study is based on the structural sensitivity analysis and result from the algebraic properties of the incidence matrices (Lefebvre, 2003, Lefebvre, et al., 2003). As a consequence, the resulting diagnosers provide delayed alarms, in the sense that they may require the occurrence of intermediate events in order to detect and isolate the firing of "failure" transitions. Another article is proposed by the authors to IFAC 05 that concerns "immediate" diagnosers that detect and isolate the firing of "failure" transitions immediately after the occurrence of the faults (Lefebvre, 2004).

The paper is divided into 5 sections. The section 2 gives an overview of the relevant literature. The section 3 concerns the use of PN models for diagnosability of DES. The section 4 is about the structural sensitivity. The section 5 is devoted to the diagnosability characterisation. 


\section{RELEVANT LITERATURE}

The objective of the diagnosis problem is to identify the occurrence and type of faults based on the observable traces generated by the system. Faults diagnosis in the context of DES was first formulated with automata (Sampath, et al., 1995). The diagnosability of the system is based on the study of the undetermined cycles of the associated diagnoser. The previous results have been extended to PN (Ushio, et al.,1998). For the PN under consideration, it is assumed that some places are observable, other places are not, whereas all the transitions are not observable in the sense that their occurrences are not known. Moreover the PN are live and safe, and there does not exist any unobservable cycle. The firing of the transitions is estimated by the changes of marking at observable places. In (Chung, et al. 2003) some transitions are assumed to be observable in the sense that their firings can be measured. Asynchronous diagnosis by means of hidden state history reconstruction obtained from alarm observations was also investigated (Benveniste, et al., 2003). This approach relies on PN unfoldings and event structures that are related via some causality relationships. As a consequence, diagnosis is performed by a distributed architecture of supervisors. At last, let us mention that the problem of diagnosis is related to the problem of sensor selection that was investigated for discrete event systems as an optimisation problem (Debouk, et al., 1999) with NP complexity (Yoo, et al., 2002).

Our approach is based neither on marking trees nor on PN unfolding. In fact, marking is not concerned and we focus on causality relationships and directed paths provided by the digraph structure of $\mathrm{PN}$.

\section{PN MODELS FOR THE DIAGNOSIS OF DES}

\subsection{Background notions on Petri nets}

A Petri net $(\mathrm{PN})$ with $n$ places and $p$ transitions is defined as $<P, T$, Pre, Post, $M_{0}>$ where $P=\left\{P_{i}\right\}_{i=1, \ldots, n}$ is a not empty finite set of places, $T=\left\{T_{j}\right\}_{j=1, \ldots, p}$ is a not empty finite set of transitions, such that $P \cap T=\varnothing$ (David, et al., 1992, Murata, 1989). IN is defined as the set of integer numbers. Pre: $P \times T \rightarrow I N$ is the preincidence application: Pre $\left(P_{i}, T_{j}\right)$ is the weight of the arc from place $P_{i}$ to transition $T_{j}$ and $W_{P R}=\left(w^{P R}\right)$ ${ }_{i=1, \ldots, n, j=1, \ldots, p} \in I N^{n \times p}$ with $w^{P R}{ }_{i j}=\operatorname{Pre}\left(P_{i}, T_{j}\right)$ is the pre-incidence matrix. Post: $P \times T \rightarrow I N$ is the postincidence application: Post $\left(P_{i}, T_{j}\right)$ is the weight of the arc from transition $T_{j}$ to place $P_{i}$ and $W_{P O}=\left(w^{P O}{ }_{i j}\right)$ $i=1, \ldots, n, j=1, \ldots, p \in I N^{n \times p}$ with $w^{P O}{ }_{i j}=\operatorname{Post}\left(P_{i}, T_{j}\right)$ is the post-incidence matrix. The PN incidence matrix $W$ is defined as $W=W_{P O}-W_{P R} \in I N^{n \times p}$. Let us also define $M=\left(m_{i}\right)_{i=1, \ldots, n} \in I N^{n}$ as the marking vector and $M_{0} \in I N^{n}$ as the initial marking vector. ${ }^{\circ} T_{j}\left(\operatorname{resp} T_{j}{ }^{\circ}\right)$ stands for the pre-set (resp. post-set) places of $T_{j}$. A firing sequence is defined as an ordered serie of transitions that are successively fired from marking $M$ to marking $M^{\prime}$. Such a sequence is represented by its characteristic vector $X=\left(x_{j}\right)_{j=1, \ldots, p} \in I N^{p}$ where $x_{j}$ stands for the number of $T_{j}$ firings. The marking $M^{\prime}$ is related to the marking $M$ and to the firing sequence $X$ according to (1):

$$
M^{\prime}=M+W \cdot X
$$

\subsection{Subnets and conflicts}

A subnet PN' of PN with $n$ ' places and $p$ ' transitions is defined as $\left\langle P^{\prime}, T^{\prime}\right.$, Pre', Post', $M^{\prime}{ }_{0}>$ where $P^{\prime} \subset P$ is a subset of $P$ and $T^{\prime} \subset T$ is a subset of $T$. Pre': $P^{\prime} \times T^{\prime} \rightarrow I N$ and Post' $: P^{\prime} \times T^{\prime} \rightarrow I N$ are respectively the restrictions of the pre and post-incidence applications limited to the sets $P^{\prime}$ and $T^{\prime} . M_{0}^{\prime} \in I N^{n^{\prime}}$ is the initial marking vector of PN'. In that sense, a subnet is defined for any subset of places $P^{\prime}=\left\{P_{i}^{\prime}\right\}_{i=1, \ldots, n}$, and transitions $T^{\prime}=\left\{T_{j}^{\prime}\right\}_{j=1, \ldots, p^{\prime}}$. The marking vector

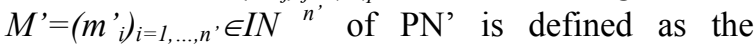
projection $M^{\prime}=D^{\prime} . M$ of the vector $M$ over the set $P^{\prime}$ with $D^{\prime} \in\{0,1\}^{n^{\prime} \times n}$. The same holds for the firing

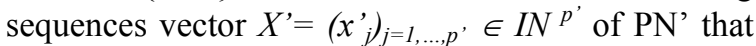
is defined as the projection $X^{\prime}=Q^{\prime} \cdot X$ of the vector $X$ over the set $T^{\prime}$ with $Q^{\prime} \in\{0,1\}^{p^{\prime} \times p}$. The incidence matrix $W^{\prime}$ of $\mathrm{PN}^{\prime}$ is defined in the same way as $W$. When two transitions $T_{j}$ and $T_{j}$, have a common place $P_{i}$ in the pre-set, the PN has a structural conflict. Such a conflict can be considered as a subnet PN' with $P^{\prime}=\left\{P_{i}\right\}$ and $T^{\prime}=\left\{P_{i}^{\circ}\right\}$.

\subsection{Diagnosability with $P N$}

In order to decide the diagnosability of a given system as well as to perform on line diagnosis with PN models, some additional notations are introduced. A label $L \in \Delta=\{N\} \cup \Delta_{F}$ is associated to each transition. $L=N$ is interpreted as a normal behaviour and $\Delta_{F}=\left\{F_{k}\right\}, k=1, \ldots m$ is the set of failure labels (i.e. $L=F_{k}$ means that a failure of type $k$ occurs). The set $T$ of PN transitions is divided into two parts: "normal" transitions and "failure" transitions: $T=T_{N}$ $\cup T_{F}$, where $T_{F}=T_{F 1} \cup \ldots \cup T_{F m}$ is the set of different types of failures. "Normal" transitions and "failure" transitions appear usually in structural conflicts: considering a given normal state, the system may evolve according to a "normal" behaviour by firing a "normal" transition or according to a faulty behaviour by firing a "failure" transition. At the same time, $T$ is also divided into observable transitions and unobservable ones $T=T_{O}$ $\mathcal{U} T_{U}$, and failures transitions are assumed to be unobservable: $T_{F} \cap T_{O}=\varnothing$. At last, the set $P$ of PN places is also divided into observable places and unobservable ones $P=P_{O} \cup P_{U}$. The state of a PN model-based diagnoser (Ushio, et al., 1998, Chung, et 
al., 2003) consists of pairs of marking and label. When some places and some transitions are unobservable, undetermined cycles may occur. The determination of these cycles requires the construction of the observable marking tree. This approach is behavioural in the sense that it is based on the analysis of the state evolution. On the contrary, our approach takes into consideration the digraph structure of $\mathrm{PN}$ to provide structural information not depending on the state evolution. To work out the marking tree is not necessary. One can also notice that CR and DP investigation is not depending on the initial marking. No assumption is required concerning the safety and liveness of the PN models.

Figure1 shows the PN example we will use throughout this paper with $P=\left\{P_{1}, P_{2}, P_{3}, P_{4}, P_{5}\right\}$ and $T=\left\{T_{1}, T_{2}, T_{3}, T_{4}, T_{5}, T_{6}, T_{7}\right\}$ (Chung et al. 2003). The transitions $T_{1}$ and $T_{5}$ represent failure events $F_{1}$ and $F_{5}$. The set of observable places is $P_{O}$ $=\left\{P_{1}, P_{4}, P_{5}\right\}$ the set of unobservable places is given by $P_{U}=\left\{P_{2}, P_{3}\right\}$ (grey circles in figure 1 ). The set of observable transitions is $T_{O}=\left\{T_{2}, T_{3}, T_{4}\right\}$, the set of unobservable ones is given by $T_{U}=\left\{T_{1}, T_{5}, T_{6}, T_{7}\right\}$.

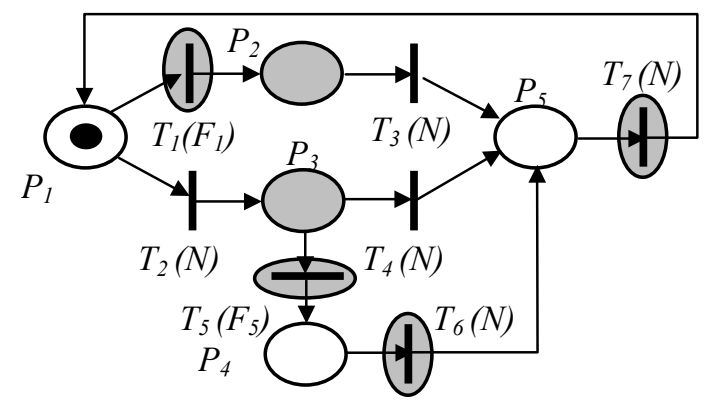

Fig. 1: PN example

\section{STRUCTURAL SENSITIVITY IN PN MODELS}

The structural sensitivity investigates the causality relationships (CR) and directed paths (DP) expressed by the pre and post incidence matrices (Lefebvre, 2003, Lefebvre, et al,. 2003). The CR is helpful to decide if the marking of a given node (place or transition) depends or not on the firing of a $F_{k}$ transition (i.e. the occurrence of a failure of type $k$ ). The DP gives an additive information to decide if such a dependence is direct or not.

Considering again the PN example given in figure 1, DP and CR can be intuitively introduced. A token in $P_{1}$ can fire the transition $T_{2}$ to move to place $P_{3}$, and then fire the transition $T_{4}$ or $T_{5}$ to move respectively to place $P_{5}$ or $P_{4}$. Thus, there exists two DP of length 1 place from $T_{2}$ to $P_{5}$ and from $T_{2}$ to $P_{4}$. There also exists two DP of length 1 place from $T_{2}$ to $T_{3}$ and from $T_{2}$ to $T_{4}$. In the same time, a token that fires $T_{2}$, cannot move directly to $P_{2}$, but can fire consecutively $T_{3}, T_{7}$ and $T_{1}$ to move to $P_{2}$. Thus, there exists a DP of length 3 places from $T_{2}$ to $P_{2}$. But the firing of $T_{2}$ will influence directly the marking of $P_{l}$ and then the firing of $T_{l}$. Thus two CR of length 1 place exists respectively from $T_{2}$ to $T_{1}$ and from $T_{2}$ to $P_{2}$.

\subsection{Causality relationships}

A CR exists from transition $T_{k}$ to place $P_{i}$ (resp. transition $T_{j}$ ) if the firing of $T_{k}$ could yield a deviation of the $P_{i}$ marking (resp. $T_{j}$ firing) from its expected value. The minimal CR-rank from $T_{k}$ to $P_{i}$, refereed as $C R\left(P_{i}, T_{k}\right)$, and from $T_{k}$ to $T_{j}$, refereed as $C R\left(T_{j}\right.$, $T_{k}$ ), are obtained according to the pre and post incidence matrices (Lefebvre 2003):

$$
\begin{aligned}
& C R\left(P_{i}, T_{k}\right)=\min _{\mathrm{r} \notin \mathbb{N} \in \phi}\left(C_{i}^{T}\left(\left(W_{P R}+W_{P O}\right) \cdot\left(W_{P R}\right)^{T}\right)^{r} \cdot\left(W_{P R}+W_{P O}\right) \cdot B_{k} \neq 0\right) \\
& C R\left(T_{j}, T_{k}\right)=\min _{\mathrm{r} \in\{\mathbb{N} \cup \infty\}}\left(B_{j}^{T}\left(\left(W_{P R}\right)^{T} \cdot\left(W_{P R}+W_{P O}\right)\right)^{r} \cdot B_{k} \neq 0\right)
\end{aligned}
$$

with $B_{k}=\left(b_{j}^{k}\right) \in\{0,1\}^{p}$ such that $b_{j}^{k}=0$ if $k \neq j$ and $b^{k}{ }_{k}=1$ and $C_{i}=\left(c_{j}^{i}\right) \in\{0,1\}^{n}$ such that $c_{j}^{i}=0$ if $i \neq$ $j$ and $c_{i}^{i}=1$. The CR - rank can be understood as the minimal number of places in the causality relationship from $T_{k}$ to $P_{i}$ or $T_{j}$. When no causality relationship exists, the CR-rank equals infinity.

Let us define $C R_{P T}=\left(C R\left(P_{i}, T_{k}\right)\right)_{i=1, \ldots, n, k=1, \ldots, p} \in\{I N$ $\left.\cup_{\infty}\right\}^{n x p}$ as the $C R$ matrix of the places $P_{i} \in P$ with respect to the transitions $T_{k} \in T$ and $C R_{T T}=\left(C R\left(T_{j}\right.\right.$, $\left.\left.T_{k}\right)\right)_{j=1, \ldots, p, k=1, \ldots, n} \in\{I N \cup \infty\}^{p \times n}$ as the $C R$ matrix of the transitions $T_{j} \in T$ with respect to the transitions $T_{k}$ $\in T$. Let us notice that the CR can not be obtained using the usual incidence matrix $W=W_{P 0}-W_{P R}$ instead of $W_{P 0}+W_{P R}$ because selfloops are ignored with the incidence matrix in the CR calculation.

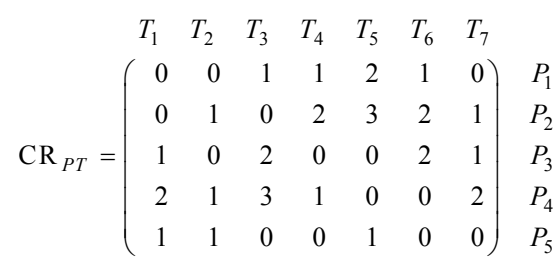

As an example, the $C R$ matrix of the places for the $\mathrm{PN}$ in figure 1 is given by (3). Any place is sensitive with respect to all transitions. For example $C R_{P T}\left(P_{2}, T_{2}\right)=1$ (the labelled $\mathrm{CR}$ is given by $\left.T_{2} P_{1} T_{1} P_{2}\right)$ and $C R_{P T}\left(P_{2}, T_{5}\right)=3$ (the labelled $\mathrm{CR}$ is given by $T_{5} P_{4} T_{6} P_{5} T_{7} P_{1} T_{1} P_{2}$ ).

\subsection{Directed paths}

A DP exists from transition $T_{k}$ to place $P_{i}$ (resp. transition $T_{j}$ ) if a token is able to move from $T_{k}$ to $P_{i}$ (resp. $T_{j}$ ). A DP between two nodes is also a CR but a CR between two nodes is not necessary a DP. The minimal DP-rank from $T_{k}$ to $P_{i}$ is refereed as $\operatorname{DP}\left(P_{i}\right.$, 
$T_{k}$ ) and the minimal DP-rank from $T_{k}$ to transition $T_{j}$ is refereed as $\operatorname{DP}\left(T_{j}, T_{k}\right)$. The following results hold (Lefebvre, 2003):

$$
\begin{aligned}
& D P\left(P_{i}, T_{k}\right)=\min _{\mathrm{r} \in\{\mathbb{N} \cup \text { oo }\}}\left(C_{i}^{T}\left(W_{P O} \cdot\left(W_{P R}\right)^{T}\right)^{r} \cdot W_{P O} \cdot B_{k} \neq 0\right) \\
& D P\left(T_{j}, T_{k}\right)=\min _{\mathrm{r} \in\{\mathbb{N} \cup 00\}}\left(B_{j}^{T}\left(\left(W_{P R}\right)^{T} \cdot W_{P O}\right)^{r} \cdot B_{k} \neq 0\right)
\end{aligned}
$$

As previously, let us define $D P_{P T}=\left(D P\left(P_{i}, T_{k}\right)\right)_{i=1, \ldots, n \text {, }}$ $k=1, \ldots, p \in\{I N \cup \infty\}^{n x p}$ as the $D P$ matrix of the places $P_{i}$ $\in T$ with respect to the transitions $T_{k} \in T$ and $D P_{T T}=$ $\left(D P\left(T_{j}, T_{k}\right)\right)_{j=1, \ldots, p, k=1, \ldots, n} \in\{I N \cup \infty\}^{p x n}$ as the $D P$ matrix of the transitions $T_{j} \in T$ with respect to the transitions $T_{k} \in T$. Let us notice that the $C R$ and $D P$ matrices can be considered as an extension of the transitive matrix (Liu, et al., 1999).

For the PN example in figure 1, $D P_{P T}\left(P_{2}, T_{2}\right)=3$ (the labelled DP is given by $\left.T_{2} P_{3} T_{3} P_{5} T_{7} P_{1} T_{1} P_{2}\right)$, $D P_{P T}\left(P_{2}, T_{5}\right)=3$ (the labelled $D P$ is given by $\left.T_{5} P_{4} T_{6} P_{5} T_{7} P_{1} T_{1} P_{2}\right)$.

$$
D P_{P T}=\left(\begin{array}{ccccccc}
T_{1} & T_{2} & T_{3} & T_{4} & T_{5} & T_{6} & T_{7} \\
0 & 2 & 1 & 1 & 2 & 1 & 0 \\
0 & 3 & 2 & 2 & 3 & 2 & 1 \\
3 & 0 & 2 & 2 & 3 & 2 & 1 \\
4 & 1 & 3 & 3 & 0 & 3 & 2 \\
1 & 1 & 0 & 0 & 1 & 0 & 2
\end{array}\right) P_{1} P_{2} P_{5}
$$

\subsection{Subnets sensitivity}

The structural sensitivity analysis can be extended to subnets. For this purpose, let us consider $P N^{\prime}$ as a subnet of $P N$. There exists a CR (resp. DP) from transition $T_{k}$ to $P N^{\prime}$ if there exists a node $N^{\prime} \in P N^{\prime}$, with a CR (resp. DP) from $T_{k}$ to $N^{\prime}$. The causality relationships for $P N^{\prime}$ is characterised by the projection of the matrices $C R_{P T}$ and $C R_{T T}$ (resp. $D P_{P T}$ and $D P_{T T}$ ) over the subnet $P N^{\prime}$ :

$$
\begin{aligned}
& C R\left(P N^{\prime}\right)=\left(\begin{array}{cc}
D^{\prime} & 0 \\
0 & Q^{\prime}
\end{array}\right) \cdot\left(\begin{array}{l}
C R_{P T} \\
C R_{T T}
\end{array}\right) \in\{I N \cup \infty\}^{\left(n^{\prime}+p^{\prime}\right) \times p} \\
& D P\left(P N^{\prime}\right)=\left(\begin{array}{cc}
D^{\prime} & 0 \\
0 & Q^{\prime}
\end{array}\right) \cdot\left(\begin{array}{l}
D P_{P T} \\
D P_{T T}
\end{array}\right) \in\{I N \cup \infty\}^{\left(n^{\prime}+p^{\prime}\right) \times p}
\end{aligned}
$$

\section{DIAGNOSABILITY OF PN MODELS}

The structural sensitivity is helpful to decide the diagnosability of a system modeled by $\mathrm{PN}$, in the sense that it provides in a systematic way the CR and DP between a "failure" transition and another node of PN. In the following, the influence and dependence areas of "failure" transitions are studied in order to evaluate the information provided by the set of observable places and transitions.

\subsection{Influence and dependence areas}

The set $I_{C R}\left(T_{k}\right)$ of nodes that are CR-sensitive with respect to the transition $T_{k}$ is called the $\mathrm{CR}$ influence area of $T_{k}$. This area is a subnet of $P N$ defined as $I_{C R}\left(T_{k}\right)=<P_{I C R}\left(T_{k}\right), T_{I C R}\left(T_{k}\right), \operatorname{Pre}_{I C R}\left(T_{k}\right)$, $\operatorname{Post}_{I C R}\left(T_{k}\right)>$ where $P_{I C R}\left(T_{k}\right) \subset P$ is the set of places $P_{i}$ such that a CR exists from $T_{k}$ to $P_{i}$ (i.e. $C R\left(P_{i}, T_{k}\right)<$ $\infty), T_{I C R}\left(T_{k}\right) \subset T$ is the set of transitions $T_{j}$ such that a CR exists from $T_{k}$ to $T_{j}$ (i.e. $C R\left(T_{j}, T_{k}\right)<\infty$ ), $\operatorname{Pre}_{I C R}\left(T_{k}\right)$ and $\operatorname{Post}_{I C R}\left(T_{k}\right)$ are the restrictions of the pre - incidence and post - incidence applications limited to the sets $P_{I C R}\left(T_{k}\right)$ and $T_{I C R}\left(T_{k}\right)$. The DP influence area $I_{D P}\left(T_{k}\right)$ is defined in a similar way.

We can also define the CR - dependence area of the node $N$. The set $T_{D C R}(N)$ of transitions that are likely to influence the node $N$ through a causality relationship is called the CR - dependence area of $N$. The DP - dependence area $T_{D D P}(N)$ is defined in a similar way. The characterisation of the sets $I_{C R}\left(T_{k}\right)$, $I_{D P}\left(T_{k}\right), T_{D C R}(N)$ and $T_{D D P}(N)$, results from the CR and DP matrices according to table 1 (Lefebvre et al., 2003).

$\underline{\text { Table } 1: \text { Influence and dependence areas }}$

\begin{tabular}{lll}
\hline \multicolumn{1}{c}{$\boldsymbol{C R}$} & \multicolumn{1}{c}{$\boldsymbol{D P}$} \\
\hline$P_{I . .}\left(T_{k}\right)$ & $\begin{array}{l}\text { position of the finite } \\
\text { entries of the } k^{\text {th }} \\
\text { column of } C R_{P T}\end{array}$ & $\begin{array}{l}\text { position of the finite } \\
\text { entries of the } k^{\text {th }} \\
\text { column of } D P_{P T}\end{array}$ \\
\hline$T_{I . .}\left(T_{k}\right)$ & $\begin{array}{l}\text { position of the finite } \\
\text { entries of the } k^{\text {th }} \\
\text { column of } C R_{T T}\end{array}$ & $\begin{array}{l}\text { position of the finite } \\
\text { entries of the } k^{\text {th }} \\
\text { column of } D P_{T T}\end{array}$ \\
\hline$T_{D . .}\left(P_{i}\right)$ & $\begin{array}{l}\text { position of the finite } \\
\text { entries of the } i^{\text {th }} \text { row } \\
\text { of } C R_{P T}\end{array}$ & $\begin{array}{l}\text { position of the finite } \\
\text { entries of the } i^{\text {th }} \text { row } \\
\text { of } D P_{P T}\end{array}$ \\
\hline$T_{D . .}\left(T_{j}\right)$ & $\begin{array}{l}\text { position of the finite } \\
\text { entries of the } j \\
\text { of } C R_{T T} \text { row }\end{array}$ & $\begin{array}{l}\text { position of the finite } \\
\text { entries of the } j^{\text {th }} \text { row } \\
\text { of } D P_{T T}\end{array}$ \\
\hline
\end{tabular}

Let us consider again the $\mathrm{PN}$ in figure 1. From the matrices $C R_{P T}$ and $D P_{P T}$ given by (3) and (5) one can first notice that $C R_{P T}\left(P, T_{1}\right)=\left(\begin{array}{lllll}0 & 0 & 1 & 2 & 1\end{array}\right)^{\mathrm{T}}$ and $D P_{P T}\left(P, T_{1}\right)=\left(\begin{array}{lllll}2 & 0 & 3 & 4 & 1\end{array}\right)^{\mathrm{T}}$. Similarly, $C R_{P T}\left(P_{2}, T\right)=\left(\begin{array}{l}0 \\ 1\end{array}\right.$

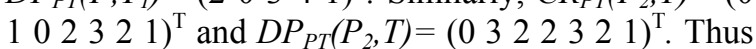
$P_{I C R}\left(T_{1}\right)=P_{I D P}\left(T_{1}\right)=\left\{P_{1}, P_{2}, P_{3}, P_{4}, P_{5}\right\}$ and $T_{D C R}\left(P_{2}\right)=P_{D D P}\left(P_{2}\right)=\left\{T_{1}, T_{2}, T_{3}, T_{4}, T_{5}, T_{6}, T_{7}\right\}$.

\subsection{Diagnosability based on DP and CR}

According to the PN models described in section 3, faults are represented by specific transitions in structural conflicts. In order to study the potential influence of the failure $F_{k}$, let us consider the subnet $\mathrm{PN}^{\prime}=\left\{{ }^{\circ} T_{k},\left({ }^{\circ} T_{k}\right)^{\circ}\right\}$ that contains all $T_{k}$ upstream places and all transitions in conflict with $T_{k}$, The following propositions hold: 
Proposition 1: Let $N \in P_{O} \cup T_{O}$. A necessary condition such that the observation of node $N$ contributes to the diagnosis of $F_{k}$ is $N \in I_{C R}\left(T_{k}\right)$.

Proof : If $N \notin I_{C R}\left(T_{k}\right)$, then the firing of $T_{k}$ does not influence the variable attached to the node $N$, and the measurement of this variable is not of interest for detection and isolation of failure $F_{k}$.

More results are obtained with the investigation of the sensitivity in the subnet $P N / T_{k}$ where the transition $T_{k}$ has been removed.

Proposition 2: Let $N \in P_{O} \cup T_{O}$. A sufficient condition to detect and isolate the firing of the failure transition $T_{k}$ with the observation of node $N$ is $N \in I_{D P}\left(T_{k}\right)$ and $T_{D D P k}(N)=\varnothing$ if $N$ is a place or $T_{D D P k}(N)=\{N\}$ if $N$ is a transition in $P N / T_{k}$.

Proof : If $N \in I_{C R}\left(T_{k}\right)$, then the firing of the failure transition $T_{k}$ influences the variable attached to the node $N$ (marking variable if $N$ is a place or firing variable if $N$ is a transition). Moreover, if $N$ is a place and $T_{D D P k}(N)=\varnothing$, then the variable attached to the node $N$ depends only on the firing of $T_{k}$. Then the measurement of this variable is sufficient for detection and isolation of failure $F_{k}$. Similarly, if $N$ is a transition and $T_{D D P k}(N)=\{N\}$, then the variable attached to the node $N$ depends only on the firings of itself and $T_{k}$. Then the measurement of this variable is also sufficient for detection and isolation of $F_{k}$.

In cases where propositions 1 and 2 cannot be applied, (i.e. if $N \in I_{D P}\left(T_{k}\right)$ and $T_{D D P k}(N) \neq \varnothing$ or $T_{D D P k}(N) \neq\{N\}$ in $P N / T_{k}$ ) then the observation of $N$ contributes but is not sufficient for the diagnosis of $F_{k}$. In this case the nodes that have to be observed at first correspond to the nodes with the smaller dependence areas. This study must be further investigated in order to combine the information obtained with the investigation of several dependence areas in order to provide minimal admissible sets of observable places as the ones resulting from immediate diagnosis.

Let us consider again the $\mathrm{PN}$ in figure 1, where transitions $T_{2}, T_{3}, \quad T_{7}$ are characterised with deterministic delays $d_{\min 2}=10, d_{\min 3}=20, d_{\min 7}=$ 30 and $T_{1}, T_{4}, T_{5}, T_{6}$ are characterised with stochastic delays given by exponential distributions $\mu_{1}=0.1$, $\mu_{4}=0.1, \mu_{5}=0.2, \mu_{6}=0.2$.

All reachable markings belong to the set $\left\{\mathrm{s}_{1}=\left(\begin{array}{lll}1 & 0 & 0\end{array}\right.\right.$ $\left.\begin{array}{lll}0 & 0\end{array}\right)^{\mathrm{T}}, \mathrm{s}_{2}=\left(\begin{array}{lllll}0 & 1 & 0 & 0 & 0\end{array}\right)^{\mathrm{T}}, \mathrm{s}_{3}=\left(\begin{array}{lllll}0 & 0 & 1 & 0 & 0\end{array}\right)^{\mathrm{T}}, \mathrm{s}_{4}=\left(\begin{array}{llll}0 & 0 & 0 & 1\end{array}\right)$ $\left.0)^{\mathrm{T}}, \mathrm{s}_{5}=\left(\begin{array}{lllll}0 & 0 & 0 & 0 & 1\end{array}\right)^{\mathrm{T}}\right\}$ and all observable markings belong to $\left\{s_{0 O}=\left(\begin{array}{lll}0 & 0 & 0\end{array}\right)^{\mathrm{T}}, \mathrm{s}_{1 \mathrm{O}}=\left(\begin{array}{lll}1 & 0 & 0\end{array}\right)^{\mathrm{T}}, \mathrm{s}_{4 \mathrm{O}}=\left(\begin{array}{ll}0 & 1\end{array}\right.\right.$ $\left.0)^{\mathrm{T}}, \mathrm{s}_{5 \mathrm{O}}=\left(\begin{array}{lll}0 & 0 & 1\end{array}\right)^{\mathrm{T}}\right\}$. The figure $2 \mathrm{a}$ shows a possible trajectory in the marking space according to the given firing sequence $X=T_{2} T_{4} T_{7} \underline{T}_{1} T_{3} T_{7} \underline{T}_{1} T_{3} T_{7} \underline{T}_{1}$ $T_{3} T_{7} \underline{T}_{1} T_{3} T_{7} \underline{T}_{1} T_{3} T_{7} T_{2} \underline{T}_{5} T_{6}$ where $5 F_{1}$ - failures and $1 F_{5}$ - failure occur consecutively (faults are underlined). The figure $2 \mathrm{~b}$ shows the observable part of this marking trajectory according to the same firing sequence $X$. Let us notice that the observable part of the marking trajectory can result not only from the actual firing sequence $X$ but also from another corrupted sequence: for instance, $X^{\prime}=T_{2} T_{4}$ $T_{7} T_{2} T_{3} T_{7} T_{2} T_{3} T_{7} T_{2} T_{3} T_{7} T_{2} T_{3} T_{7} T_{2} T_{3} T_{7} T_{2} \underline{T}_{5} T_{6}$ where only $1 F_{5}$ - failure occurs. As a consequence, the observation of the observable part of the marking trajectory does not provide enough information to detect and isolate directly the faults $F_{l}$ and $F_{5}$ and the diagnosis problem must be solved.
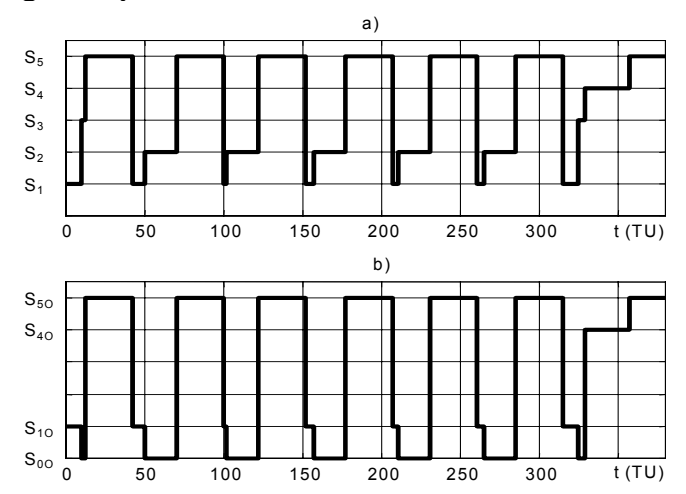

Fig. 2: a) Marking trajectory b) Observable part of the marking trajectory

The sensitivity analysis is useful to provide efficient diagnosers without building the marking tree. By working out the matrix $C R_{P T}$ (equation 3 ) one can first notice that $P_{I C R}\left(T_{1}\right)=\left\{P_{1}, P_{2}, P_{3}, P_{4}, P_{5}\right\}$ and $T_{I C R}\left(T_{1}\right)=\left\{T_{1}, T_{2}, T_{3}, T_{4}, T_{5}, T_{6}, T_{7}\right\}$. The observation of each place and each transition contributes to the diagnosability of the system.

By working out the matrices $D P_{P T}$ and $D P_{T T}$ in $P N / T_{1}$ (equation 7) and $P N / T_{5}$ one can also notice that $T_{D D P I}\left(P_{2}\right)=T_{D D P 5}\left(P_{4}\right)=\varnothing, T_{D D P I}\left(T_{3}\right)=\left\{T_{3}\right\}$ and $T_{D D P 5}\left(T_{6}\right)=\left\{T_{6}\right\}$. A diagnoser of $F_{1}$ and $F_{5}$ can be obtained with the observation of the couples of nodes $\left\{T_{3}, T_{6}\right\}$, or $\left\{P_{2}, P_{4}\right\}$ or $\left\{T_{3}, P_{4}\right\}$ or $\left\{P_{2}, T_{6}\right\}$. According to the sets of unobservable nodes $T_{U}$ and $P_{U}$, the unique admissible diagnoser is based on the observation of the nodes $\left\{T_{3}, P_{4}\right\}$. Such a diagnoser detects and isolates the faults according to the measurement and analysis of the observable firing sequences and observable markings. The delay between the occurrence of the failures $F_{1}$ and the fault detection correspond to the duration $d_{\min 1}=10$ TU (figure $3 \mathrm{a}$ ). The detection of the $F_{5}$ fault is immediate (figure $3 b$ ).

The proposed diagnoser has better performances (detection and isolation of faults $F_{1}$ and $F_{5}$ ) and require less information (observation of only 2 nodes) in comparison with the one proposed by Chung et al. who suggest to use $P_{O}=\left\{P_{1}, P_{4}, P_{5}\right\}$ and $T_{O}=\left\{T_{3}\right\}$ in order to detect $F_{1}$. Moreover, our approach does not require the construction of the marking tree. 


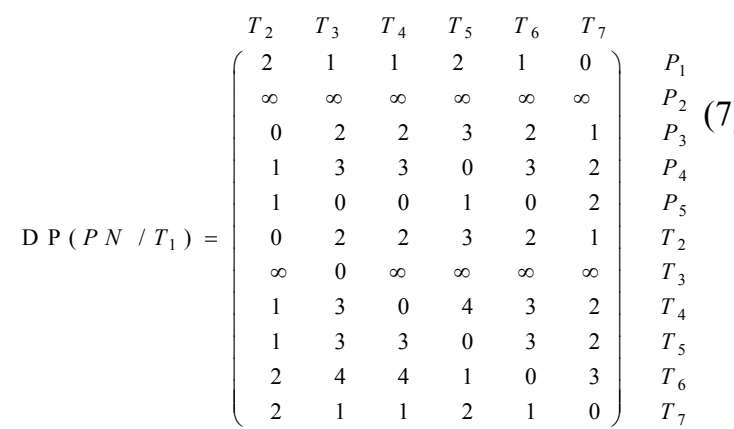

a)

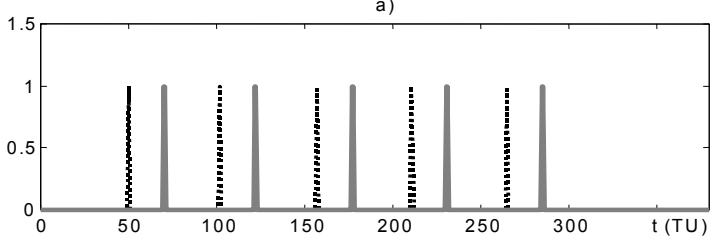

b)

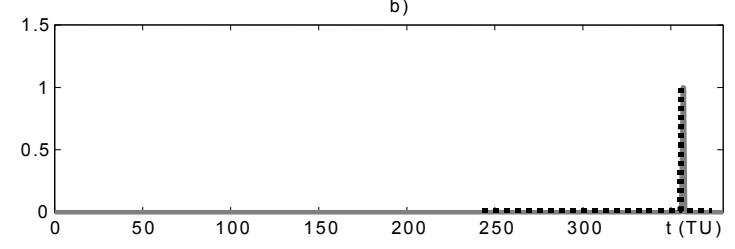

Fig.3. a) Detection of fault $F_{1}$ b) Detection of fault $F_{2}$ (measurements of the $T_{6}$ or $T_{3}$ firings in full line, faults occurrence in dotted line)

\section{CONCLUSIONS}

This paper has proposed some structural results concerning the sensitivity analysis of PN. CR and DP have been investigated in a systematic way. Influence and dependence areas of failure transitions were obtained for PN models of DES with faulty behaviours. The diagnosability of the considered systems has been obtained as a consequence. The main advantage of our approach is to decide in many cases the diagnosability without working out the observable marking tree. In some cases the diagnosability is not decidable, but in all cases our approach is helpful to build the minimal set of nodes to be observed.

Our perspectives are to investigate further the $C R$ and DP for diagnosis issues and to provide a structural solution when the observation of several nodes is required. Moreover this work takes part in our study about monitoring and safe control of DES and hybrid dynamical systems (Zaytoon, et al. 1998) modelled with PN. The use of CR and DP will also be developed for observability and controllability issues.

\section{REFERENCES}

Alcaraz-Mejia M., Lopez-Mellado E., RamirezTrevino A., Rivera-Rangel I. (2003). Petri net based fault diagnosis of DES, Proc. IEEE-SMC03, pp. 4730-4735, Washington, USA.

Benveniste A., Fabre F., Jard C., Haar S. (2003). Diagnosis of asynchronous discrete event systems, a net unfolding approach. IEEE-TAC, vol.48, no.5.

Cassandras C.G. (1993). Discrete event systems: modeling and performances analysis, Aksen Ass. Inc. Pub.

Chung S.L, Wu C.C., Jeng M. (2003). Failure diagnosis: a case study on modeling and analysis by Petri nets, Proc. IEEE-SMC03, pp. 2727-2732, Washington, USA.

David R., Alla H. (1992). Petri nets and grafcet tools for modelling discrete events systems, Prent. Hall, London.

Debouk R., Lafortune S., Teneketzis D. (1999). On An Optimization Problem In Sensor Selection, IEEE Conf. on Decision and Control, Phoenix , Arizona , December, 7-10.

Giua A., Seatzy C. (2002). Observability of place / transition nets, IEEE-TAC, vol.47, no.9, pp.14241437.

Krogh, B.H.; Holloway, L.E. (1991). Synthesis of Feedback Control Logic for Discrete Manufacturing Systems, Automatica, vol. 27, no. 4, pp. 641-651.

Lefebvre D. (2003). Sensibilité paramétrique des réseaux de Petri continus à vitesse variable, JESA, vol. 37 , no 1 , pp. $83-108$.

Lefebvre D, Delherm C. (2003). Structural sensitivity for the conflicts analysis in Petri nets, IEEE - SMC03, 7-10 october, Washington, USA,.

Lefebvre D. (2004). Admissible sets of observables places for immediate diagnosis of faulty behaviours with PN models, submit. to IFAC 05.

Liu J., Itoh Y., Miyazawa I., Sekiguchi K. (1999). A research on Petri net properties using transitive matrix, IEEE-SMC99, vol. 1, pp 888-893, Tokyo, Japan.

Murata T. (1989). Petri nets: properties, analysis and applications, Proc. IEEE, vol.77, no. 4, pp541-580.

Sampath M., Sengupta R., Lafortune S., Sinnamohideen K., Teneketzis D. (1995). Diagnosibility of discrete event systems, IEEE$T A C$, vol. 40, no.9, pp. 1555- 1575.

Ushio T., Onishi I., Okuda K. (1998). Fault detection based on Petri net models with faulty behaviours, IEEE - SMC, pp 113-118.

Yoo T., Lafortune S. (2002). NP-Completeness of Sensor Selection Problems Arising In Partiallyobserved Discrete-Event Systems, IEEE TAC, vol. 47, no. 9, pp. 1495-1499.

Zaytoon J., (editor) (1998). Hybrid dynamical systems, APII - JESA, vol 32, n 9-10. 